Original Research Paper

\title{
Optimal Meshing of Structured Boundary Domains in Numerical Analyses
}

\author{
${ }^{1}$ Komla Kpogli, ${ }^{1}$ Sibiri Wourè-Nadiri Bayor, \\ ${ }^{1}$ Ayité Senah Akoda Ajavon, ${ }^{2}$ Kokou Tcharie and ${ }^{3}$ Arnulf Kost \\ ${ }^{I}$ Departement of Electrical Engineering,Ecole Nationale Supérieure d'Ingénieurs (ENSI), Université de LOME, Togo \\ ${ }^{2}$ Departement of Mathematics, Faculté des Sciences (FDS), Université de LOME, Togo \\ ${ }^{3}$ Lehrstuhl Allgemeine Elektrotechnik und Numerische Feldberechung, \\ TU Cottbus, Postfach 101344, D-03013 Cottbus, Germany
}

\author{
Article history \\ Received: 23-08-2017 \\ Revised: 23-09-2017 \\ Accepted: 21-10-2017 \\ Corresponding Author: \\ Sibiri Wourè-Nadiri Bayor \\ Departement of Electrical \\ Engineering, Ecole Nationale \\ Supérieure d'Ingénieurs \\ (ENSI), Université de LOME, \\ Togo \\ Email: bayores1@yahoo.fr
}

\begin{abstract}
The analysis of a practical problem using numerical methods such as Finite Element Method (FEM) or Boundary Element Method (BEM) involves the subdivision of the space occupied by a physical system (named domain) or its boundary into sub-domains or sub-boundaries called elements. This task, known as mesh generation or domain-discretization, is no more trivial if domains of real or industrial problems involving shape irregularities, various different materials which may be non-linear or anisotropic; are to be taken into account. In this paper an easy mesh technique considering even domains, which are tiresome handlebar by Computer Aided Design (CAD) Software, has been proposed to achieve efficient and suitable meshes to minimize the computer storage requirements, the computation time; and to improve the accuracy of the results during numerical Analyses. The basic elements adopted in mesh are d-Simplexes.
\end{abstract}

Keywords: Mesh-Generation, View-Point, Optimal-Point, d-Simplex, Shape- Regulation

\section{Introduction}

Any numerical analysis relying on methods such as Finite Difference Method (FDM), Finite Element Method (FEM) and Boundary Element Method (BEM), involves the subdivision of the space occupied by a physical system into appropriate elements. This task known as mesh generation or domain-discretization is the first and the most important part in any numerical method process, because the manner in which the domain is meshed will affect the computer storage requirements, the computation time, and accuracy of the numerical results (Burnett, 1987; Jin, 1993). In this process of partitioning a domain into a mesh of elements, the question of the optimum way of connecting $n$ points in a d-dimensional space to form elements of acceptable shape arises (CGAL, 2015). In fact the problem raised here is a technique of subdividing a domain $\Omega$ (assuming it has polyhedral boundaries) into a set $\bar{\Omega}$ of triangles or rectangles (two-dimensional space), tetrahedrons, triangular prisms or rectangular bricks (three-dimensional space) which have to respond to the following conditions:

C1) The sum of all elements of $\bar{\Omega}$ is equal to $\Omega$

C2) The intersection of two elements of $\bar{\Omega}$ is a point, an edge, a facet or empty (non-overlapping condition)
C3) All the elements should be made close to equilateral as possible or avoid generating elements having a small inner angle; the reason is they increase the solution error. It can be read in (Jin, 1993) that the error of the finite element solution is inversely proportional to the sine of the smallest inner angle

C4) A greater degree of freedom density (number elements) in areas of interest and lesser degree of freedom density elsewhere in the region $\Omega$

The main aim of the technique proposed in this paper was to achieve a mesh for real objects which are tiresome to be reproduced by CAD Software (AutoCAD and COMSOL for examples) and create their Geometries for numerical analysis purposes: In fact without any knowledge of CAD Software, just through mathematical real body modelling, object boundary structuring, d-Simplex Shape Regulation and the philosophy of object oriented approach, mesh can be obtained by a given list of points describing the considered domain. This objective has been obviously reached by using structured boundary approaches, and as examples, models and their meshes have been proposed for practical FEM and BEM analyses in (Komla and Kost, 2003; De Castro, 2010; Korman et al., 2014). 


\section{Method}

\section{Modelling of Real Body}

In numerical methods (FDM, BEM, FEM) a space occupied by a physical system is considered as finite and closed. It's most of the time complex and composed of several sub-regions according to the physical properties of the system in question. Say $\Omega$ such a d-dimensional real body, it's approximated to a sum of closed d-dimensional sub-regions (polyhedrons in 3-dimensional -, polygons in two-dimensional - and segments of line in 1-dimensional space) $\Omega_{j}, j=1,2, \ldots, M$, so that:

$\Omega \approx \cup_{j=l}^{M} \Omega_{j}, \forall i, j, 1 \leq M, \Gamma_{i j}=\Omega_{i} \cap \Omega_{j j}$

Where:

For $\mathrm{i} \neq \mathrm{j}$,

$\Omega_{i}$ and $\Omega_{j}=$ sub-regions

$\Gamma_{i j}$, = interface or external-face of $\Omega_{i}$ and $\Omega_{j}$ : May be empty, a point, a segment of line or a polygon.

The structure of the closed boundary $\Gamma=\partial \Omega$ of the region $\Omega$ depends on boundary loads and the behaviour of the physical properties in this area. After approximation (1) $\Gamma$ is consequently equal to a sum of sub-boundaries (polygons in three-dimensional -, segments of line in twodimensional - and points in 1-dimensional case) $\Gamma_{j}, j=1$, $2, \ldots, N$, so that:

$$
\Gamma \approx \cup_{j=1}^{N} \Gamma_{j}, \forall i, j, 1 \leq N, E_{i j}=\Gamma_{i} \cap \Gamma_{j}
$$

Where:

For $\mathrm{i} \neq j$,

$\Gamma_{i}$ and $\Gamma_{j}=$ sub-boundaries

$E_{i j}=$ intersection of two sub-boundaries: may be empty, a point or segment of line

The border $\partial \Gamma$ of any $\Gamma_{i}$, is assumed to be a sum of entities (segments of line in three-dimensional - and points in two-dimensional case) $E_{i}, \mathrm{i}=1,2, \ldots, K$, so that:

$$
\begin{aligned}
& \partial \Gamma \approx \cup_{i=1}^{K} E_{i}, \forall i, j, i \neq j \\
& P_{i j}=E_{i} \cap E_{j}=\varnothing \text { or point, and } \\
& E_{i}=\left[P_{m} P_{n}\right], m \neq n
\end{aligned}
$$

Where:

For $i \neq j$,

$P_{i j}=$ the intersection of two elements $E_{i}$ and $E_{j}$ of $\partial \Gamma$

$P_{m}$ and $P_{n}=$ Border points of the entities $E_{i}$.

\section{Object Oriented Approach}

During the analysis of the problem through approximations ((1) - (4)), the following geometrical objects have been mathematically identified to fit the modeling concepts of real body adopted in this paper:

- d-Point: A d-dimensional space point characterised by its coordinates, representing nodes or vertices of elements in mesh of the region $\Omega$

- d-Edge: A portion of d-dimensional space straight line bordered by two d-Point objects, representing edges of elements in mesh of the region $\Omega$

- p-Face: A portion of d-dimensional planar surface bordered by sets of interconnected d-Edge objects, which are indeed closed lines and will be referred to as c-Line objects in the rest of this paper. In fact a pFace object is considered as a face of a polyhedron as defined in equation (1) and represents entities defined in equation (2) in three-dimensional case. A c-Line object in this case denotes the entity $\partial \Gamma$ as defined in equation (3)

- $\quad$-Volume: A closed polyhedral three-dimensional sub-region bordered by a set of interconnected pFace objects, forming a closed three-dimensional surface which will be referred to as c-Surface object in the rest of this paper. A p-Volume object represents the closed sub-region as defined in equation (1), and its closed boundary is a c-Surface object, formed by interconnected p-Face objects

In addition to these objects some types of mesh elements have been defined as follows:

- d-Simplex: An envelope of d+1 d-Point objects in d-dimensional space (Fig. 1(a) and (b)), building mathematically a system of $(\mathrm{d}+1)$ points with a nonzero determinant. A d-Simplex object is actually a known geometrical object (a tetrahedron in threedimensional -, a triangle in two-dimensional - and a segment of line in one-dimensional case), which $d+1$ facets are (d-1)-Simplexes named

- $\quad$ s-Facet objects (triangles in three-dimensional -, dEdges in two-dimensional - and d-Points in onedimensional case):

- d-Element: Denotes a type of elements in which the $\mathrm{d}$-dimensional region $\Omega$ is meshed. A d-Element object is actually a known geometrical object which can be obtained by merging d-Simplexes

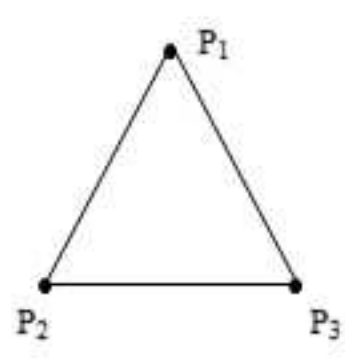

(a)

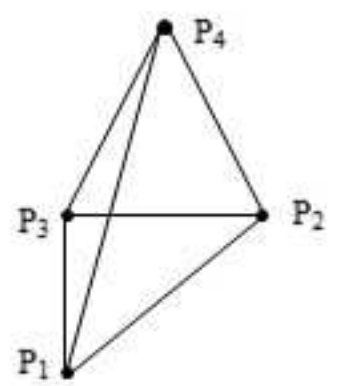

(b)
Fig. 1. (a) 2-Simplex (b) 3-Simplex 
The determinant of the d-simplex $=\left\{P_{1}, P_{2}, \ldots, P_{d+1}\right\}$ may be written as:

$\operatorname{det}(d-S i m p l e x)=\left|\begin{array}{ccccc}p_{2}^{1}-p_{1}^{1} & \ldots & p_{i}^{1}-p_{1}^{1} & \ldots & p_{d+1}^{1}-p_{1}^{1} \\ p_{2}^{2}-p_{1}^{2} & \ldots & p_{i}^{2}-p_{1}^{2} & \ldots & p_{d+1}^{2}-p_{1}^{2} \\ \cdot & & \cdot & & \cdot \\ \cdot & & \cdot & & \cdot \\ \cdot & & \cdot & & \cdot \\ p_{2}^{d}-p_{1}^{d} & \ldots & p_{i}^{d}-p_{1}^{d} & \ldots & p_{d+1}^{d}-p_{1}^{d}\end{array}\right|$

Where:

For $i=1, \ldots, d+1$ and $n=1, \ldots, d$

$p_{i}=$ The d-simplex vertices

$p_{i}^{n}=$ The Cartesian co-ordinates of the $i^{\text {th }}$ point

\section{Algorithm}

Through objects identification and mesh elements definition above a practical approach of mesh generation has led to define a d-Edge, a p-Face and p-Volume objects as one-dimensional, two-dimensional and threedimensional fundamental meshing objects respectively. Consequently a d-dimensional mesh is defined in this paper as interconnected d-dimensional fundamental meshing objects discretized in d-Elements. And a d-dimensional mesh generation is fully an automatic object to object meshing technique which must be imagined as a mason who is assigned to build a house without a drawn plan by an architect. The only physical orientation given to him is a piece of land $(\Omega)$ with its border $(\Gamma)$ description and a number of conditions to observe during the construction. Fortunately, he was also given the number floors (dimension d) and the form which each room (d-Element) must have. Well, with the help of his intelligence, he starts from the first floor (with any one of the fundamental meshing objects) builds room by room, observing the conditions required and setting properties for the next room till the entire house is completed on the last floor. The whole process is step by step job realised in three stages:

- Object boundary structuring

- Object meshing into d-simplexes and d-simplexes merging to d-Elements

- Objects connecting

For simplicity and commodity p-Face - and pVolume objects are called later d-Polyhedrons; and cLines - and c-Surface objects are named d-Surfaces in two- and three-dimensional cases.

\section{Object Boundary Structuring}

In fact the connected p-Face objects of a c-Surface are orientated according to their normal vectors $\vec{n}$ and the connected d-Edges objects of a c-Line are transformed in coplanar vectors as is shown in Fig. 2.

\section{Object Meshing into d-Simplexes}

It is a fully stepwise Object Oriented Mesh generation consisting principally of three steps in two-dimensional and five steps in three-dimensional cases as is shown in Fig. 3: The first step is indeed a d-Edge object refining (Face borders regulation); the second and third stages denote a pFace objects meshing (Face mesh generation and Face elements regulation); and the fourth and fifth stages represent a p-Volume objects meshing (Volume mesh generation and Volume elements regulation).

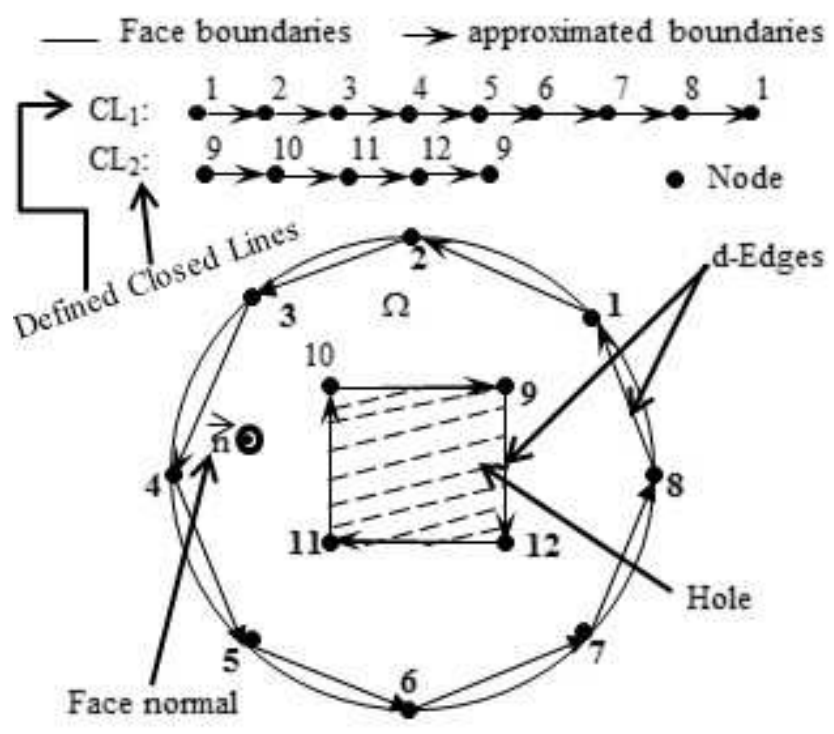

Fig. 2. Model of a p-Face defined by a circle with an inner hole 


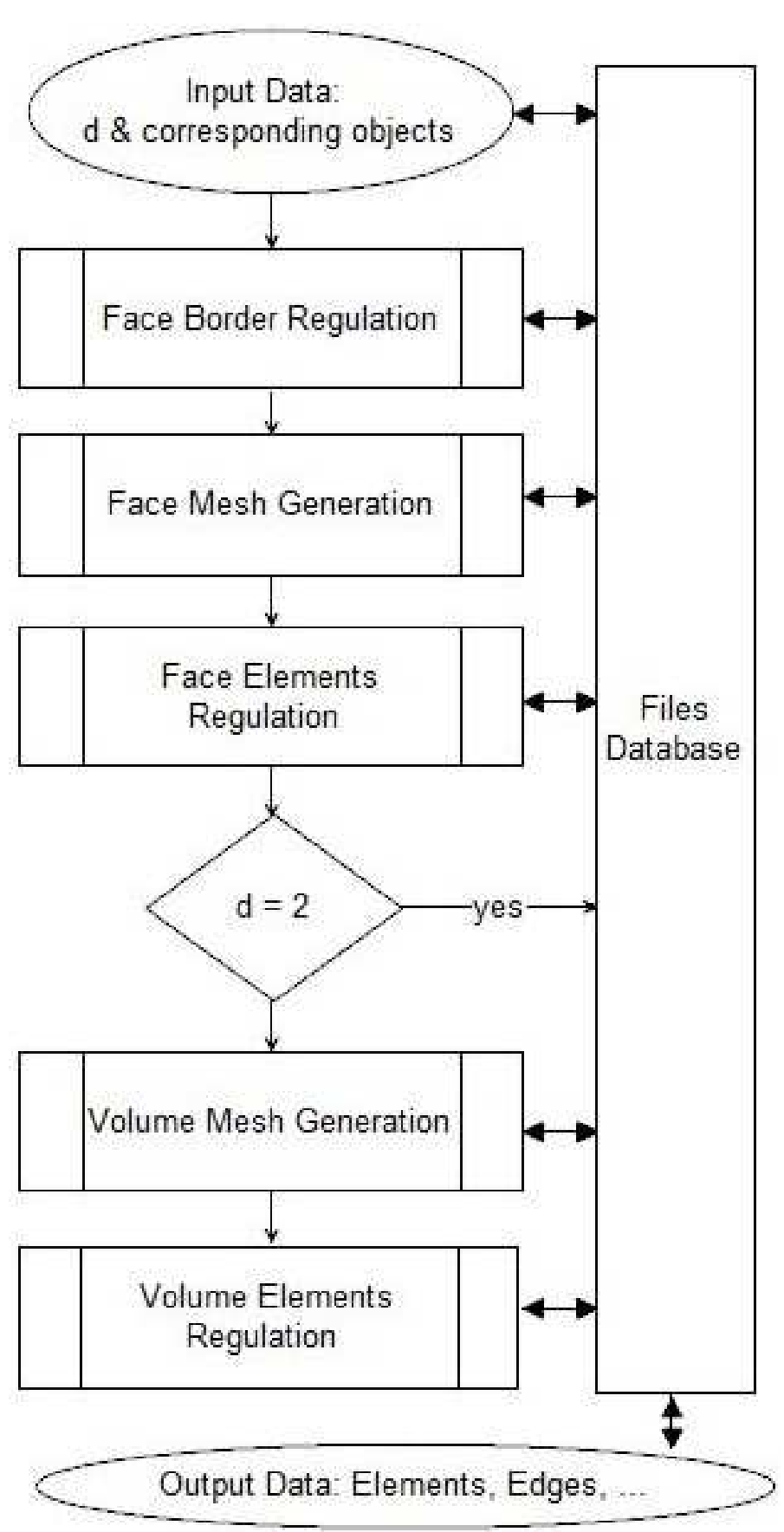

Fig. 3. Mesh object into d-Simplexes

The construction of a d-Simplex object adopted here depends on two fundamental criterions named viewpoint of s-Facet object and s-Facet object regularity:

- For a given s-Facet F lying inside a d-Polyhedron, any d-Point $\mathrm{P}$ element of this d-Polyhedron building a non-degenerated virtual d-Simplex $S=\{F, P\}$ lying inside the d-Polyhedron and satisfying the condition C2) above, is labelled a view-point of $F$
- A s-Facet object $F$ is regular if its circumscribed ( $d$ 1)-Sphere $\boldsymbol{B}_{r}$ (Fig. 4) doesn't contain any of its probable view-points

If an s-Facet object is not regular, it's resized to fit the regularity condition (for example in Fig. 4, the twodimensional s-Facet $F_{1}=\left[P_{5}, P_{6}\right]$ is regular, and s-Facet $F_{2}=\left[P_{3}, P_{4}\right]$ on the other hand is not). 


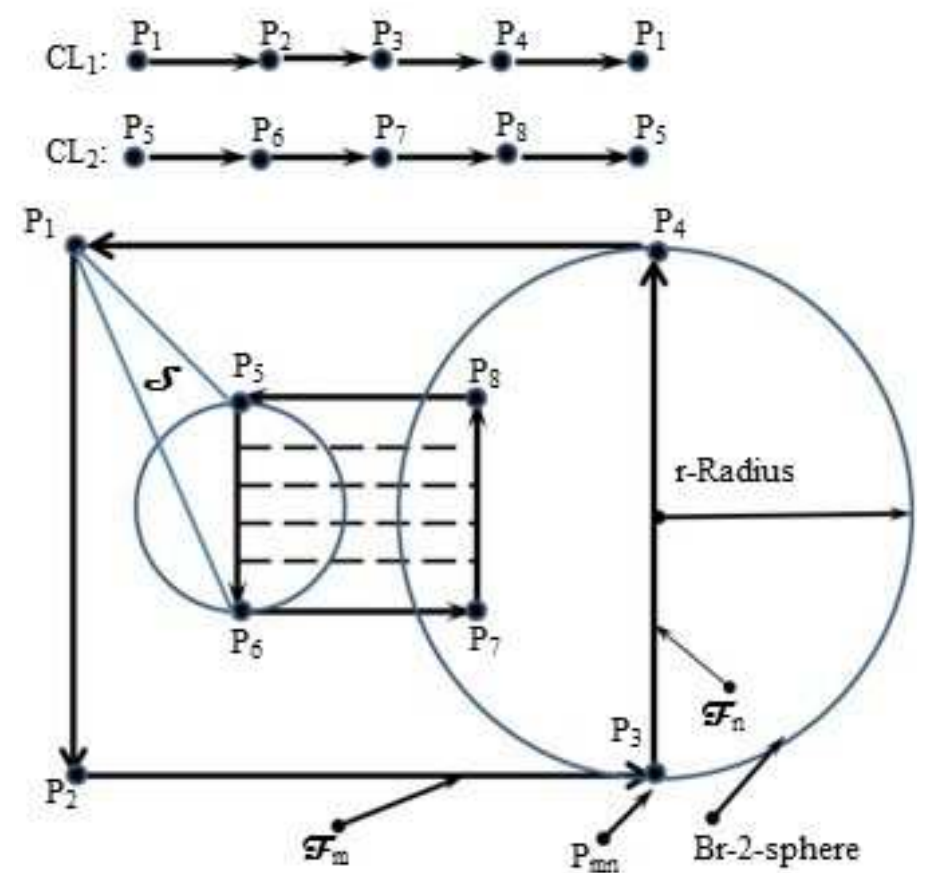

Fig. 4. Face Border Regularity check

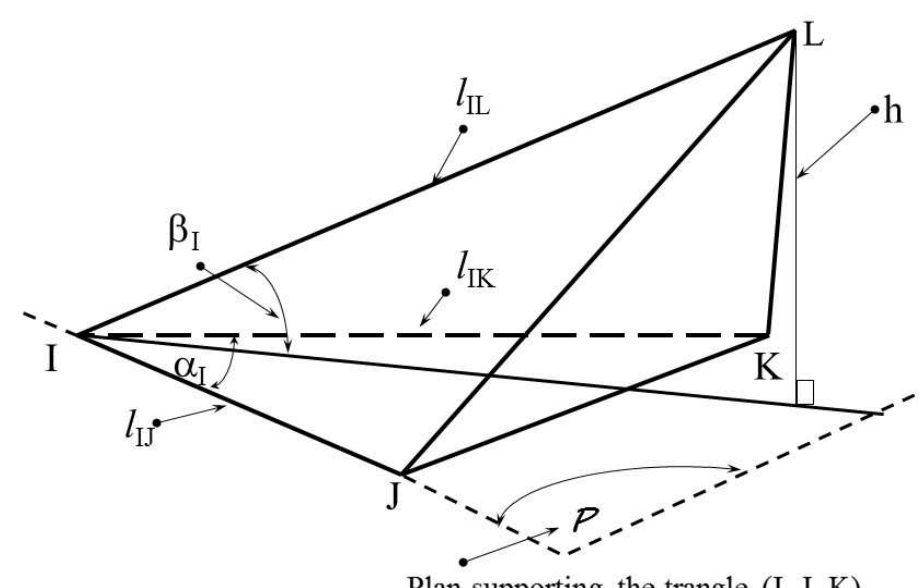

Plan supporting the trangle (I, J, K)

Fig. 5. 3-simplex

Among the view-points of an s-Facet object, the one with the best condition $\mathrm{C} 3$ ) is called the optimal point of the element.

The optimal point is determined by evaluating the following quantities at each vertex of a d-Simplex (Fig. 5):

$\sin \left(\alpha_{\mathrm{I}}\right)=\frac{2 \Delta}{l_{\mathrm{IJ}} l_{\mathrm{IK}}}$, for a triangle

$\sin \left(\beta_{\mathrm{I}}\right) \sin \left(\alpha_{\mathrm{I}}\right)=\frac{3 \mathrm{~V}}{l_{\mathrm{IJ}} l_{\mathrm{IK}} l_{\mathrm{LL}}}$, for a tetrahedron

Where:
$l_{I J}, l_{I K}$ and $l_{I L}=$ The lengths of edges starting from the vertex $I$

$\Delta=$ The area of the triangle $(I, J, K)$

$\mathrm{V}=$ The volume of the tetrahedron $(I, J, K, L)$

$\alpha_{I}$ and $\beta_{I}=$ The inner angles at the vertex $I$

The basic idea of the concept of meshing a dPolyhedron $\mathcal{D}$ into interconnected d-Simplexes satisfying the condition $\mathrm{C} 2$ ) above may be summarised as follows: Find progressively optimal points $P_{\text {opt }}$ to cover all uncovered s-Facet objects (Fig. 6) $\boldsymbol{F}_{k}, k=$ $1, \ldots, n$ Facet, lying inside the d-Polyhedron $D$, so that a mesh of d-Simplexes $S_{k}=\left\{F_{k}, P_{\text {opt }}\right\}$ is generated for $\mathcal{D}$. 


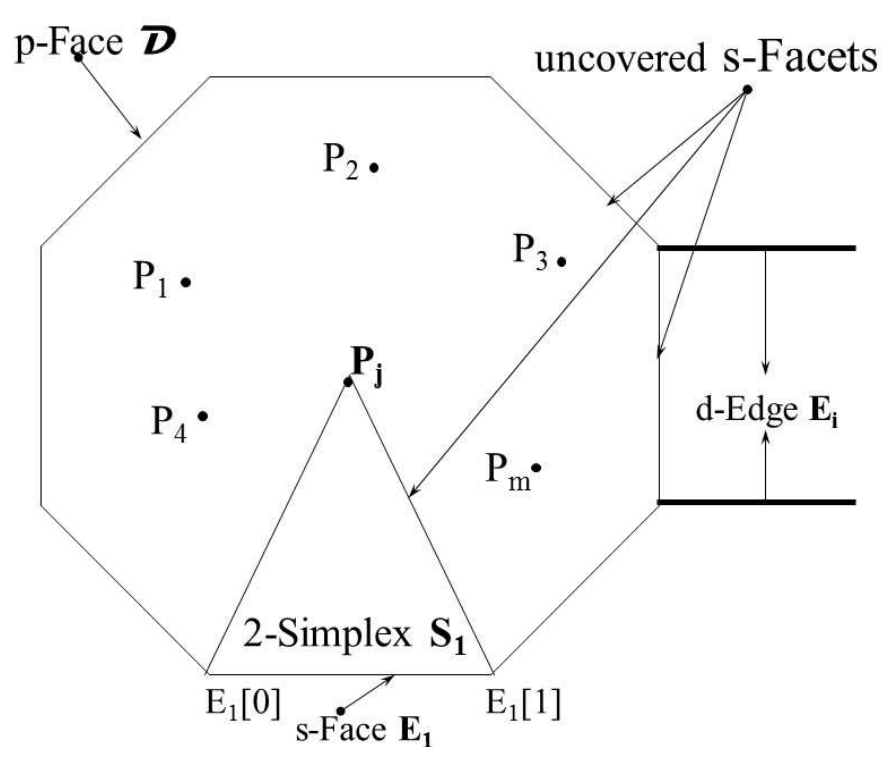

Fig. 6. p-Face Meshing in 2-Simplexes $\mathscr{F}$

At beginning of the process it is supposed that the d-Surface $\boldsymbol{B}$ of the d-Polyhedron is covered with regular s-Facet objects (Fig. 6). These elements are automatically the initial uncovered s-Facets objects $\mathscr{F}$ lying inside the d-Polyhedron. This process of meshing a given d-dimensional domain in $d$ Simplexes is generally called triangulation of the domain. The triangulation approach of a given $d-$ Polyhedron $\mathcal{D}$ in this paper is summarised in Fig. 7.

\section{Simplex Shape Regulation}

The final triangulation of $\mathcal{D}$ is obtained by controlling all the d-simplexes through regulations to satisfy the condition C3). Furthermore the aim is to bring the final mesh to a quality near the one of Delaunay Tessellation (Watson, 2006; François, 2008; Boissonnat., 2008; Geuzaine and Remacle, 2009; Brévilliers, 2008; Aichholzer et al., 2015) to ensure the regularity of the elements: Exceptions however are made only in cases of four co-cyclical - in two-dimensional and five cospherical points in three-dimensional meshes as presented in Fig. 8 and Fig. 9.

In this paper a concept of neighbour nodes position check is additionally introduced through the idea of dSimplex-face neighbour point zone. The concept is that, there is for every facet of a d-simplex a special zone, which is properly defined by the structure of it to contain a neighbour point and ensure the regularity in the region.

The neighbour point of a face of a d-Simplex e is the $n^{\text {th }}$ vertex of the neighbour element $e_{n}$ which doesn't belong to e. For example $\mathrm{N}$ is the neighbour point of the face named face-k in (Fig. 10). In general a k-zone of a face-k of a d-simplex is a half opened region k-zone bounded by the d-1 dimensional subspaces supporting the faces of the d-simplex.
In fact it is easy to see, that any point $\mathrm{N}$ in this $\mathrm{k}$-zone forms together with the face-k of the d-simplex e a nondegenerated d-simplex $e_{n}=\{$ face-k, $\mathrm{N}\}$ if the distance between face-k and the point $\mathrm{N}$ is kept reasonable. In other words the point $N$ must not be too far from and also too closed to the face-k. To be able to get an optimal $\mathrm{k}$-zone for the face-k two delimiter spheres are introduced. The first one is the circumscribed $\mathrm{d}$-sphere $B_{r}$ of the d-simplex e and the second a sphere $\mathrm{B}_{\mathrm{R}}$ which has the same centre as the $\mathrm{d}$-sphere but with radius $\mathrm{R}$ greater than the radius $r$ of the first one (Fig. 11). The face neighbour point zone is defined as:

$\mathrm{FNPZ}_{\mathrm{k}}=\mathrm{k}$-Zone $\cap \mathrm{B}_{\mathrm{r}} \cap \mathrm{B}_{\mathrm{R}}$

for a k-face of an element.

The centre $\mathrm{C}$ and the radius $\mathrm{r}$ of the circumscribed $\mathrm{d}$ sphere $B_{r}$ of each $d$-simplex in triangulation are determined by equations (9) and (10):

$$
\begin{aligned}
& \left\{\begin{array}{c}
C^{1} \\
\vdots \\
C^{d}
\end{array}\right\}=2\left[\begin{array}{ccc}
p_{1}^{1}-p_{2}^{1} & \cdots & p_{1}^{d}-p_{2}^{d} \\
\vdots & \vdots & \vdots \\
p_{1}^{1}-p_{d+1}^{1} & \cdots & p_{1}^{d}-p_{d+1}^{d}
\end{array}\right]^{-1}\left\{\begin{array}{c}
\sum_{i=1}^{d}\left(p_{1}^{i}\right)^{2}-\left(p_{2}^{i}\right)^{2} \\
\vdots \\
\sum_{i=1}^{d}\left(p_{1}^{i}\right)^{2}-\left(p_{d+1}^{i}\right)^{2}
\end{array}\right\} \\
& r^{2}=\sum_{i=1}^{d}\left(p_{1}^{i}-C^{i}\right)^{2}
\end{aligned}
$$

Where:

For $i=1, \ldots, \mathrm{d}+1$ and $\mathrm{n}=1, \ldots, d$ :

$p_{i}=$ The vertices of the d-simplex

$P_{i}^{n}=$ The Cartesian co-ordinates of the vertices

$C^{n}=$ The Cartesian co-ordinates of the centre $C$ 
The choice of the radius $\mathrm{R}$ of the sphere depends on the optimal size of the transition elements one wants to have approximately in the region of interest. Here $\mathrm{R}=\alpha \mathrm{r}, \alpha>1, \alpha \in \square$.

Now a k-face of an element is admissible if the neighbour point $\mathrm{N}$ belongs to $F N P Z_{k}$. In case the condition is not satisfied then we have to check if $N \in$ $B_{r}$ or not. If $N \in B_{r}$ then the 'swapping rule' is used to rearrange the elements see (Watson, 2006; Jänicke and Kost, 1992) otherwise a new point is generated in the $F N P Z_{k}$ for the face-k.

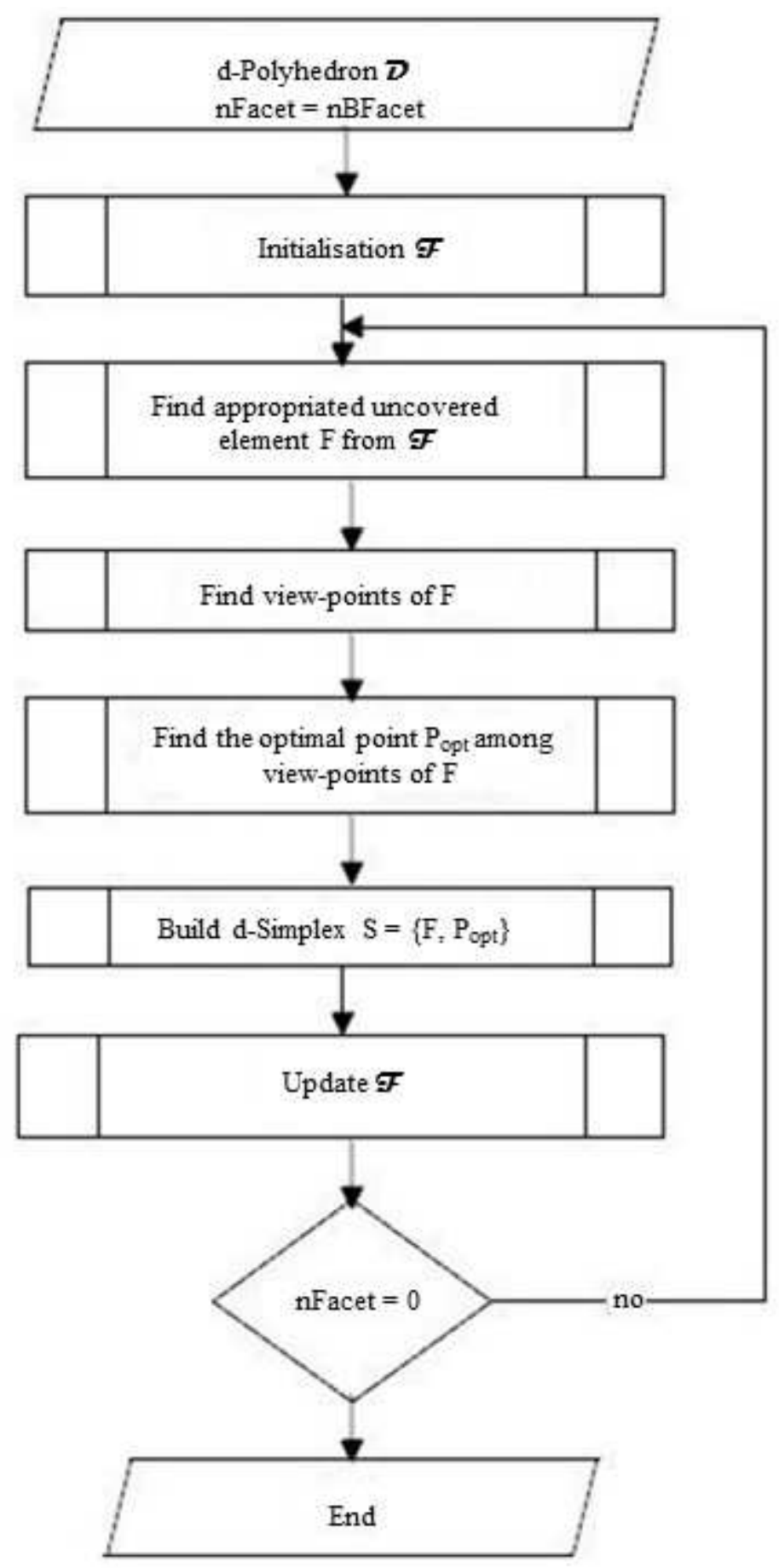

Fig. 7. Triangulation of a d-Polyhedron $\mathcal{D}$ 


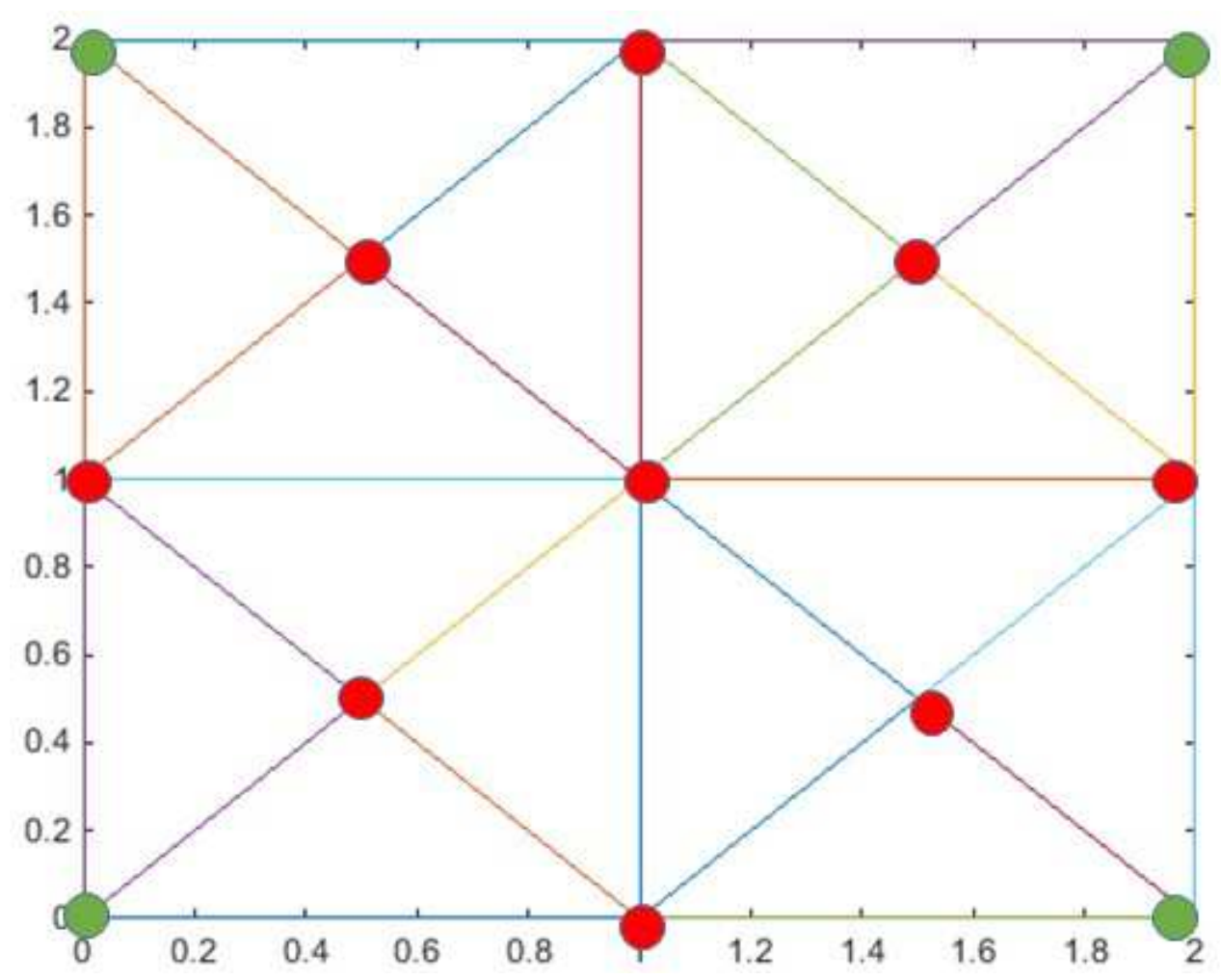

Fig. 8. Triangular mesh

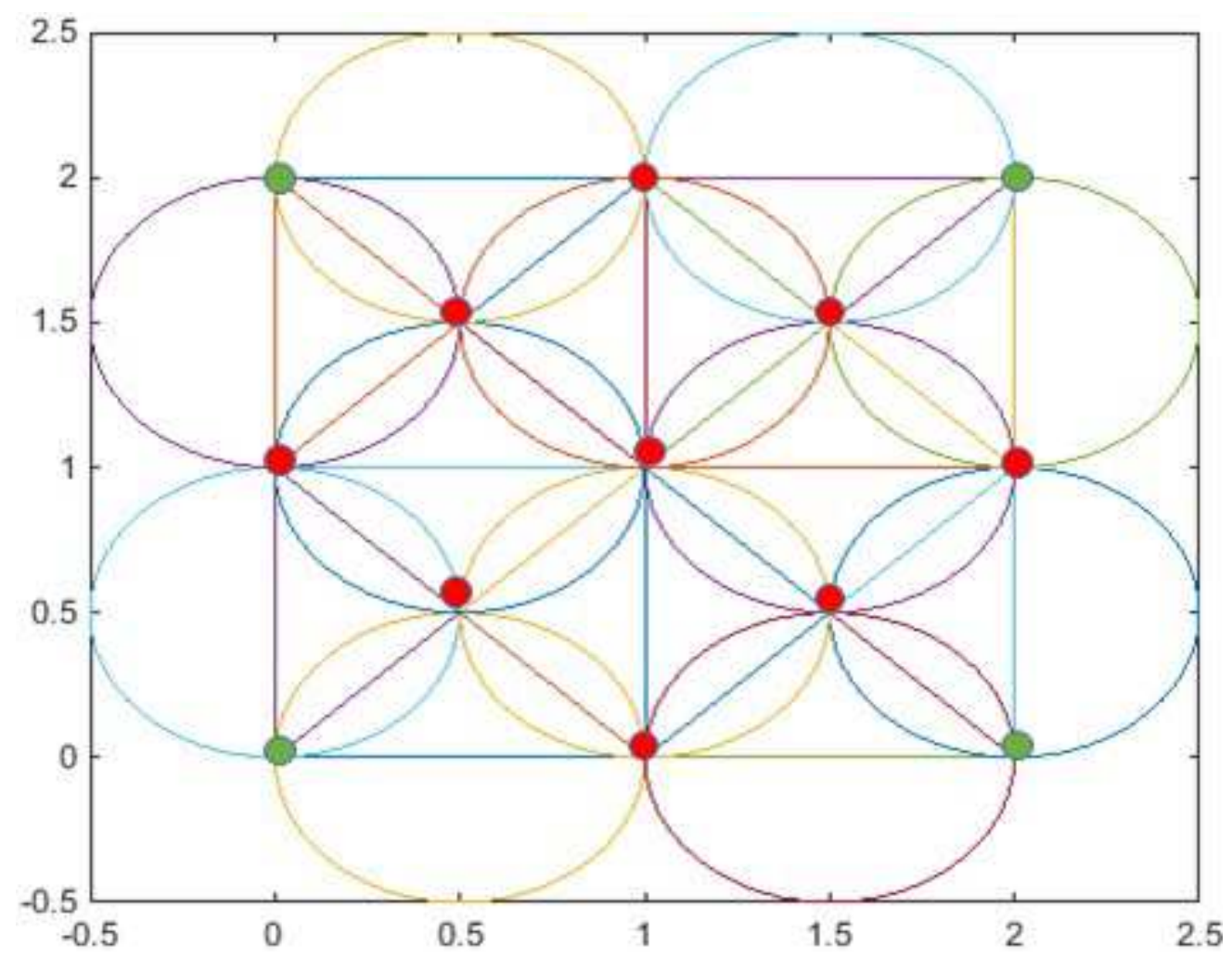

Fig. 9. Triangular mesh with four co-cyclical points 


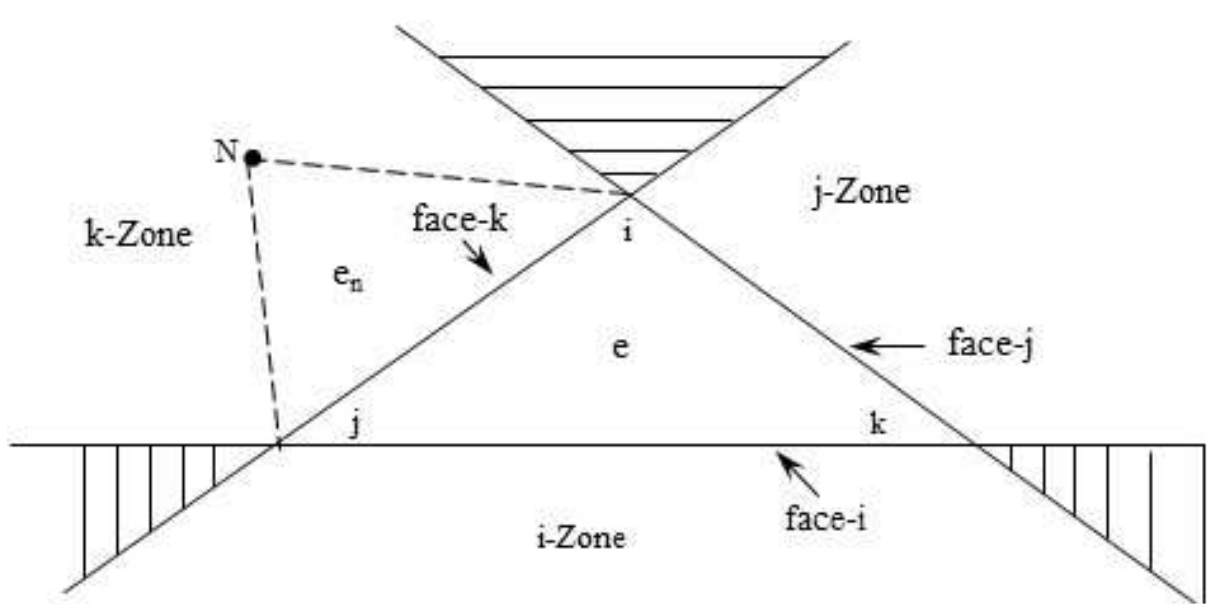

Fig. 10. Definition of acceptable region for neighbour points

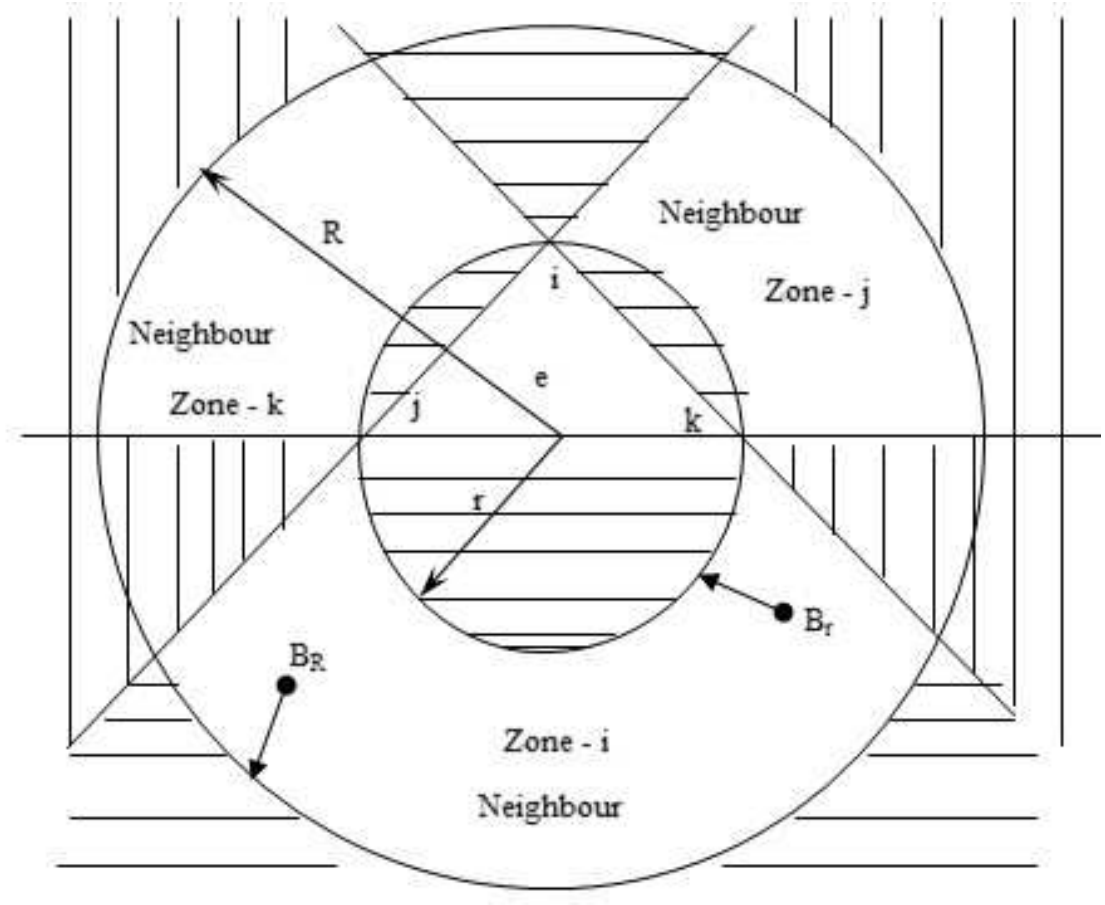

Fig. 11. Definition of acceptable region for neighbour points

\section{Results}

FEM Problem Introduced in (Webb and Forghani, 1995)

3-dimensional Mesh proposed for the FEM problem introduced by Webb and Forghani to assess the relative performance of their hierarchical tetrahedron reconsidered in (Komla and Kost, 2003): The model consists of magnetic circuit driven by a coil carrying uniform current density with a total current of $1 \mathrm{~A}$. A copper block is placed in the air gap of the magnetic circuit (Fig. 12).
TEAM-Workshops Problem No.7 (Turner, 1988)

Mesh proposed for the TEAM-Workshops problem No.7 made of Asymmetrical Conductor with Hole $(\mathrm{ACH})$ and a coil reconsidered in (Komla and Kost, 2003): It consists of a square conducting plate $(\sigma=$ $3.526 \times 10^{7} \mathrm{Sm}^{-1}$ ) with a square hole in one corner and a coil carrying sinusoidal source current density which is constant over the cross section of the coil, placed asymmetrically over the plate (Mesh: Fig. 13a, created Geometry: Fig 13b). 


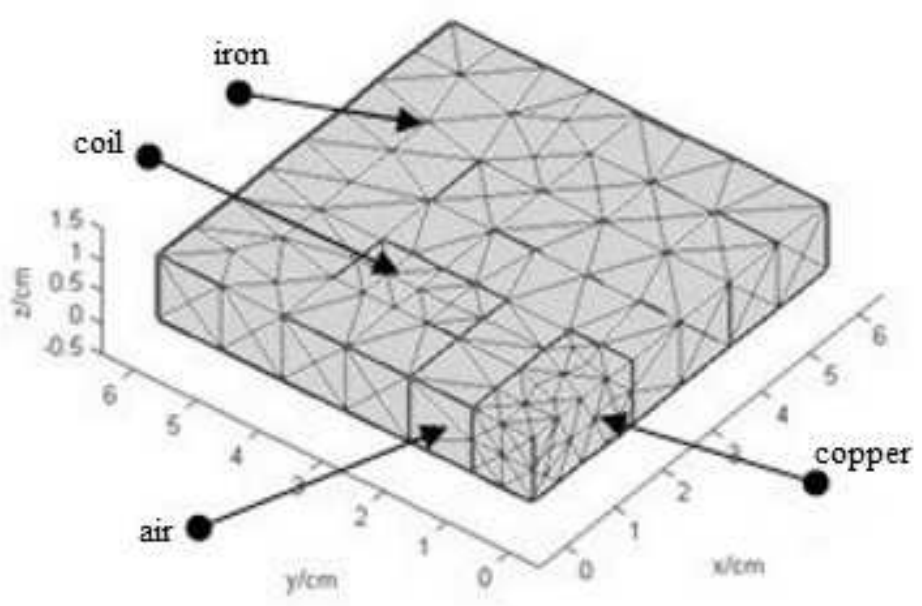

Fig. 12. 3-dimensional FEM model

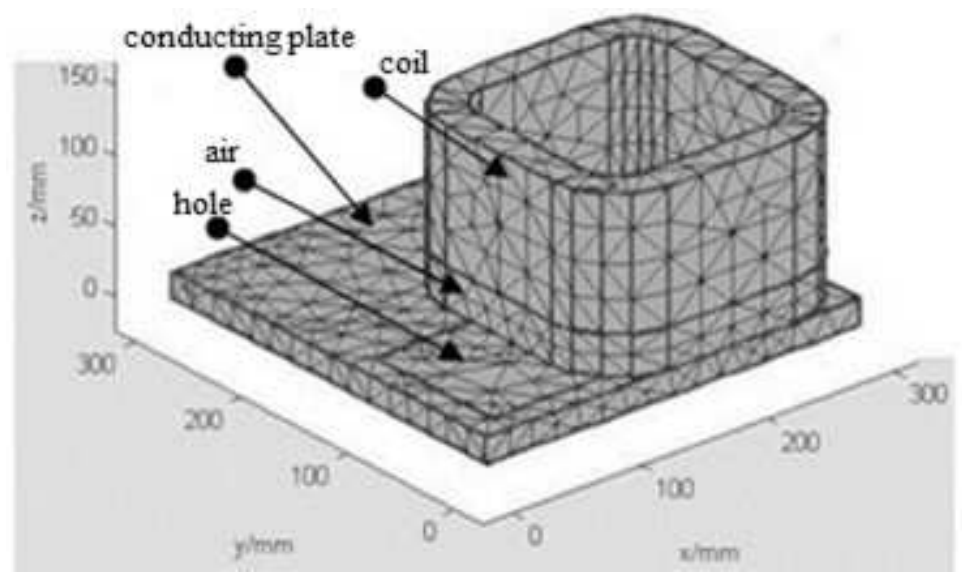

(a)

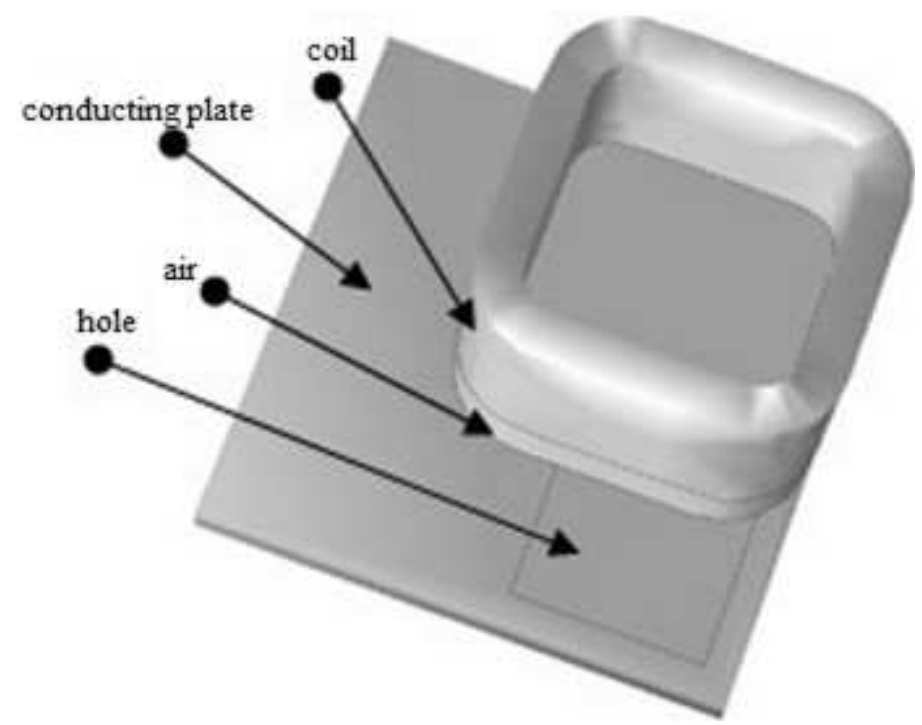

(b)

Fig. 13. (a) Conducting plate with a hole driven by a coil (b) Geometry created from mesh in Fig. 11a 


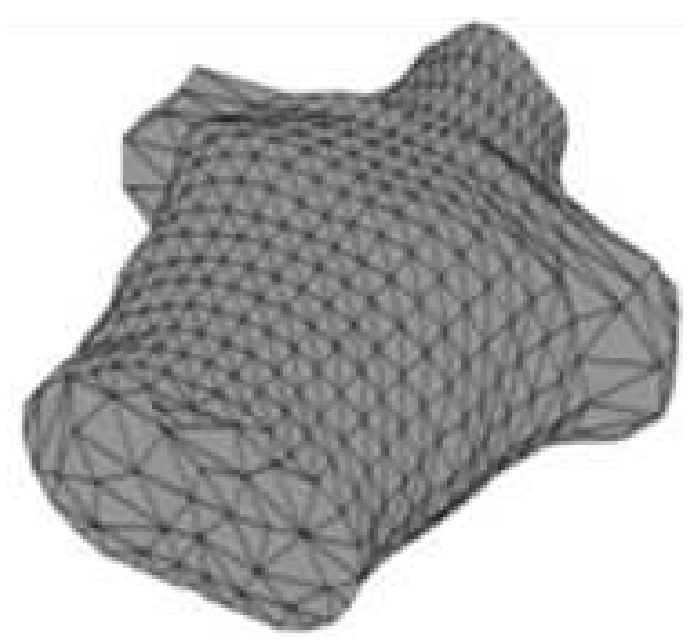

Fig. 14a. Measured thorax of a person (FEM Model)

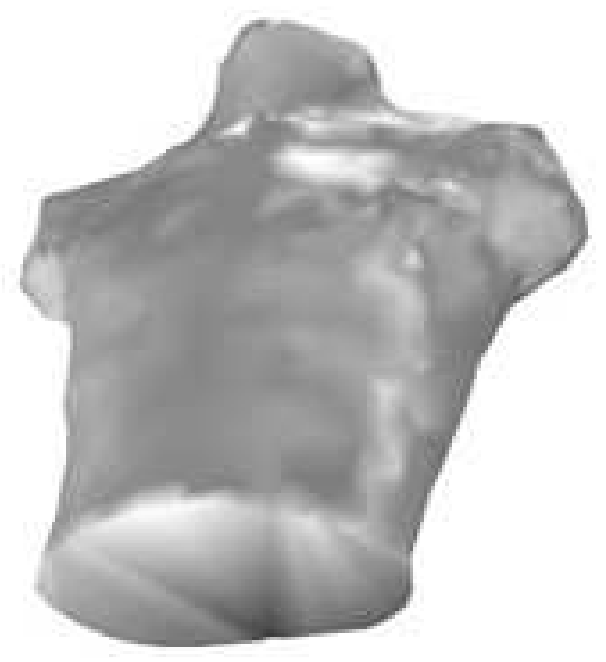

Fig. 14b. Geometry created from Mesh

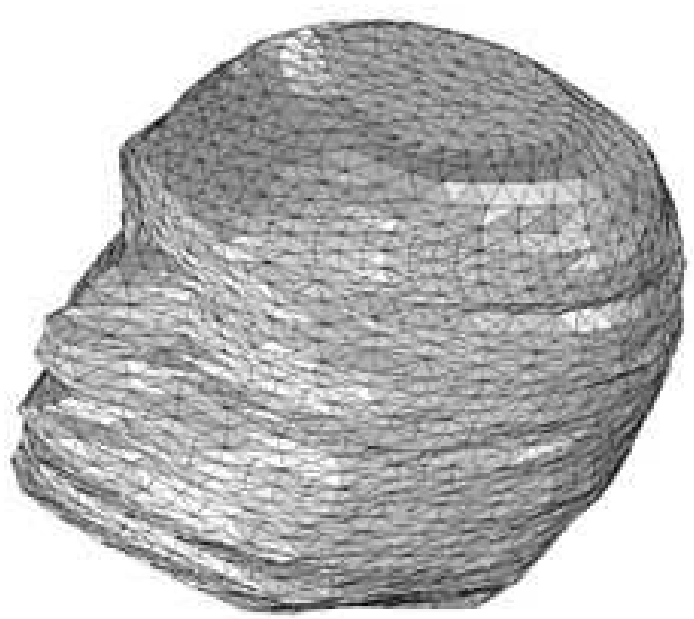

Fig. 15. Measured head of a person

\section{Measured Data from Siemens AG Berlin, Germany}

Mesh proposed for measured data from Siemens AG, Berlin (Mesh: Fig. 14a and. 15, created Geometry Fig. 14b).

\section{Conclusion}

The mesh generation, the first and the most important part in any numerical method process, has been chosen as basis of investigation in this paper. The aim was to reach a flexible and optimal mesh generator which must be able to generate a mesh containing only regular elements for any given space occupied by a physical system, if it can be approximated, according to its physical properties, as segregated sub-regions as presented in sections "Modelling of real body" and "Object oriented approach". The main goal in this paper was to provide efficient and suitable mesh for numerical analyses even where Computer Aided Design software may have difficulties to achieve a result. Through structured boundary input data successful meshes have been achieved and real objet models are created which can be used by CAD software. Practical examples have been considered to test the new mesh generator developed and some of the results have been used in (Komla and Kost, 2003) to analyze successfully Magneto-Thermal problems in Electrical Engineering on Finite Element Method (FEM) basis.

\section{Acknowledgement}

We thank Essowè Komlan ESSIZEWA, PhD., Professor in Sociolinguistics (Dean of Faculty of Arts and Languages University of Lomé, Togo) for the English Grammar checking.

\section{Author's Contributions}

Komla KPOGLI: Participated in concepts, source codes writing and contributed to the writing of the manuscript.

Sibiri Wourè-Nadiri BAYOR: Participated in concepts, coordinated the programing process and contributed to the writing of the manuscript.

Ayité Senah Akoda AJAVON: Participated in concepts and contributed to the writing of the manuscript.

Kokou TCHARIE: Participated in concepts and contributed to the writing of the manuscript.

Arnulf KOST: Participated in concepts and contributed to the writing of the manuscript.

\section{Ethics}

The present work is not published in its present form in any journal or will not be published in any journal. 


\section{References}

Aichholzer, O., W. Aigner, F. Aurenhammer, K.C. Dobiasova and B. Juttler et al., 2015. Triangulations with Circular Arcs. J. Graph Algorithms Applic, 19: 43-65. DOI: 10.7155/jgaa.00346

Boissonnat, J.D., 2008. Geometrica INRIA sophiaantipolis algorithmique des triangulations et modelisation geometrique. Ecole Normale Supérieure de Cachan.

Brévilliers, M., 2008. Construction de la triangulation de Delaunay de segments par un algorithme de flip. $\mathrm{PhD}$ Thèses of Philosophy, Université de Haute Alsace.

Burnett, D.S., 1987. Finite Element Analysis: From Concepts to Applications. 1st Edn, Addison-Wesley, ISBN: 0-201-10806-2, pp: 844.

CGAL, 2015. 3D mesh generation, user manuel. www.Doc.cgal.org

De Castro, P., 2010. Practical ways to accelerate Delaunay triangulations. PhD Thesis of Philosophy, Software Engineering [cs.SE], Université Nice Sophia Antipolis.

François, H., 2008. Nouvelles méthodes de volumes finis pour approcherdes équations aux dérivées partielles sur des maillages quelconques. CEA/DAM Ile de France Département Sciences de la Simulation et de L'Information Service Numérique Environnement et Constantes.
Geuzaine, C. and J.F. Remacle, 2009. A threedimensional finite element mesh generator with built-in pre- and post-processing facilities. Int. J. Numerical Methods Eng., 79: 1309-1331.

Jänicke, L. and A. Kost, 1992. Universal generation of an initial mesh for adaptive 3-D finite element method. IEEE Trans. Magnet., 28: 1735-1738.

Jin, J., 1993. The Finite Element Method in Electromagnetics. 1st Edn, John Wiley and Sons, New York, ISBN-10: 0471586277, pp: 464.

Komla, K. and A. Kost, 2003. Local error estimation and strategic mesh generation for time-dependent problems in electromagnetics coupled with heat conduction. IEEE Trans. Magnet., 39: 1701-1704. DOI: 10.1109/TMAG.2003.810524

Korman, M., S. Langerman, W. Mulzer, A. Pilz and B. Vogtenhuber, 2014. Minimum Dual Diameter Triangulations. Proceedings of the 30th European Workshop on Computational Geometry, Mar. 3-5, Ein-Gedi, Israel, pp: 1-4.

Turner, L.R., 1988. TEAM-workshops. Test Problems.

Watson, D.F., 2006. Computing the n-dimensional Delaunay tessellation with application to Voronoi polytopes. Department of Geology and Geophysics, Edgeworth Building, University of Sydney, NSW, Australia.

Webb, J.P. and B. Forghani, 1995. T- $\Omega$ Method using hierarchal edge elements. IEEE Proc. Sci. Meas. Technol., 142: 133-141. 